\title{
Novel mutations of FRMD7 in Chinese patients with congenital motor nystagmus
}

\author{
XIUHUA JIA $^{1 *}$, XIANG ZHU $^{2 *}$, QIGEN LI $^{1}$, XIAOYUN JIA $^{3}$, SHIQIANG LI $^{3}$ and XIANGMING GUO ${ }^{3}$ \\ ${ }^{1}$ Department of Ophthalmology, ${ }^{2}$ Department of Infectious Diseases, The Third Affiliated Hospital, \\ Sun Yat-sen University, Guangzhou, Guangdong 510630; ${ }^{3}$ State Key Laboratory of Ophthalmology, \\ Zhongshan Ophthalmic Center, Sun Yat-sen University, Guangzhou, Guangdong 510060, P.R. China
}

Received July 10, 2016; Accepted April 25, 2017

DOI: $10.3892 / \mathrm{mmr} .2017 .6824$

\begin{abstract}
The purpose of the current study was to identify novel mutations in the FRMD7 (FERM domain containing 7) gene and to characterize clinical features in Chinese patients with congenital motor nystagmus. For this purpose, 18 patients with congenital motor nystagmus were selected from the ocular genetic diseases bank of the Pediatric and Genetic Clinic of Zhongshan Ophthalmic Center (Guangdong, China). Direct sequencing was used to analyze the exons and adjacent introns of the FRMD7 gene. The heteroduplex-single strand conformation polypeptide method was used to analyze 96 unrelated normal controls and gene-screening positive patients. Slit lamp photography of the anterior segment, fundus photography, optical coherence tomography and electroretinogram were carried out to identify the clinical features of congenital motor nystagmus. The authors noted that in, 18 patients with congenital motor nystagmus, there were 7FRMD7 gene mutations (six new mutations). The screening rate was $38.89 \%$, including c.41_43delAGA (p.13-15delK); c.473T>A (p.I158N); c.605T >A (p.I202N); c.580G $>$ T (p.A194S); c.811T>A (p.C271S); c.1493insA (p.Y498X); c.57+1G $>$ A (slice mutation). There were no such mutations in the 96 normal controls. These results enriched the gene mutation spectrum of FRMD7. The authors systematically investigated the clinical phenotype of congenital motor nystagmus in a Chinese population. The study provides further evidence for clinical diagnosis and differential diagnosis and genetic counseling.
\end{abstract}

Correspondence to: Professor Xiangming Guo, State Key Laboratory of Ophthalmology, Zhongshan Ophthalmic Center, Sun Yat-sen University, 54 Xian Lie Nan Road, Guangzhou, Guangdong 510060, P.R. China

E-mail: zocguoxm@aliyun.com

${ }^{*}$ Contributed equally

Key words: FERM domain containing 7, genovariation, congenital motor nystagmus

\section{Introduction}

Congenital nystagmus $(\mathrm{CN})$ is a group of hereditary eye diseases, although the cause of the diseases has yet to be elucidated. It can be divided into sensory defective nystagmus and motor defective nystagmus according to its etiology. Sensory defective nystagmus can be originated from insufficiency image stimulus of macular region induced by the disease of anterior visual pathway, or the function loss of the fovea induced by organic diseases of macula, retina or optic nerve $(1,2)$. Usually, sensory defective nystagmus can occur in a variety of diseases, such as congenital cataracts, aniridia, Peters' anomaly, oculocutaneous albinism, achromatopsia, cone rod dystrophy, macular defects, congenital stationary night blindness, Leber congenital amaurosis and optic nerve hypoplasia $(1,2)$. Motor defective nystagmus may be originated from the central nervous system or the abnormal pathway controlling the eye movements. Patients with motor defective nystagmus don't have other ocular abnormalities $(3,4)$. Congenital motor nystagmus (CMN), also known as congenital idiopathic nystagmus, is an isolated form of nystagmus, consisting of involuntary oscillations of the eyes. It is characterized by an absence of other ocular pathologies. A variety of genetic modes, such as autosomal dominant, autosomal recessive and $\mathrm{X}$ linked, have been proven to be associated with CMN (5-8).

In 1999, two X-linked CMN loci were reported, demonstrating that this form of inheritance is also genetically heterogeneous with a locus for X-linked irregularly dominant $\mathrm{CMN}$, as reported by Kerrison et al (9) at Xq26-q27 (NYS1) and by Cabot et al (10) at Xp11.4-p11.3.8.

However, only one gene has been identified to be associated with X-linked CMN. In 2006, Tarpey et al (4) first identified nystagmus-causing mutations in the FRMD7 gene within the Xq26-q27 interval. In the present study, FRMD7 mutation analysis and detailed clinical evaluation were performed to identify novel mutations and characterize new clinical features of the Chinese population with CMN.

\section{Materials and methods}

Patients and clinical data. The patients presenting CMN were referred to the Pediatric and Genetic Clinic in the Eye 
Hospital of the Zhongshan Ophthalmic Center (Guangzhou, China). Written informed consent was obtained from each participant prior to the study. The present study was approved by the Ethics Committee of the Zhongshan Ophthalmic Center (Guangzhou, China) and was performed according to the tenets of the Declaration of Helsinki. Medical and ophthalmic histories were obtained. A complete general physical examination and detailed ophthalmological examinations, including anterior segment observation with slit-lamp microscopy, fundus photography and optical coherence tomography, were conducted to identify the clinical features of CMN.

Mutation screening. Genomic DNA was prepared from venous blood. All of the primers (Takara Bio, Inc., Otsu, Japan) for FRMD7 (Table I) were used to amplify coding exons (exon1 to exon 12 of FRMD7) and the adjacent intronic sequence of the gene (NCBI human genome build 36.3, NC_000023.10 for gDNA, NM_194277.2 for cDNA and NP_919253.1 for protein of FRMD7). The PCR reaction was performed in a thermocycler (Biometra $\mathrm{GmbH}$, Göttingen, Germany) under the following two conditions: The first was an initial denaturation at $94^{\circ} \mathrm{C}$ for $5 \mathrm{~min}$ followed by 35 to 37 cycles of $94^{\circ} \mathrm{C}$ for $30 \mathrm{sec}$, proper annealing temperature for $30 \mathrm{sec}$, and $72^{\circ} \mathrm{C}$ for $30 \mathrm{sec}$ with a final extension cycle of $72^{\circ} \mathrm{C}$ for $5 \mathrm{~min}$. The other condition was the touch down PCR program (Fig. 1). The PCR products of the exons and adjacent intronic sequences for the patients were sequenced with the ABI BigDye Terminator direct sequencing kit (version, 3.1; Applied Biosystems; Thermo Fisher Scientific, Inc., Waltham, MA, USA) according to the manufacturer's recommendations and using a 3100 sequencer (Applied Biosystems; Thermo Fisher Scientific, Inc.) confirmed by the authors. Sequencing results from patients, as well as consensus sequences of FRMD7 from the NCBI human genome database (NM_001604.3) were imported into the SeqManII program of the Lasergene Package (DNASTAR, Madison, WI, USA) and aligned to identify variations. Each mutation was confirmed by bidirectional sequencing. Mutation was named according to the nomenclature recommended by the Human Genomic Variation Society.

Determination of changes in genetic information. The nucleotide sequences containing base variation and the standard sequences from NCBI human genome database were inputted into the MapDraw program of the Lasergene package to identify the impact of amino acid coding. Protein sequences of different species were identified from the NCBI website and entered into the MegAlign program of the Lasergene package, in order to compare the protein sequences of different species and estimate the conservation of mutation sites in the ClustalW program. The differences in amino acid changes and the possible pathogenicity of the mutation were evaluated through the Blosum 62 (http://www. uky.edu/Classes/BIO/520/BIO520WWW/blosum62.htm) and PolyPhen (http://genetics.bwh.harvard.edu/pph/) (11) analytical programs respectively.

Heteroduplex single-strand conformation polymorphism (SSCP) analysis. The variations detected in the gene were further evaluated in 96 normal controls (informed consent, in accordance with the Declaration of Helsinki, was obtained from the participating individuals before the study) by using heteroduplex-SSCP analysis as previously described in literature (12-14). DNA fragments of mutation sites were PCR-amplified according to Table I. PCR products were mixed with an equal volume of gel-loading buffer (95\% formamide, $20 \mathrm{mM}$ EDTA and $0.05 \%$ bromophenol blue, $0.05 \%$ xylene cyanol) and denatured at $95^{\circ} \mathrm{C}$ for $5 \mathrm{~min}$ and immediately placed on ice for $5 \mathrm{~min}$. The samples were loaded directly onto $8 \%$ polyacrylamide gels and run $8 \mathrm{~h}$ at room temperature at $40 \mathrm{~W}$ in a solution of $0.5 \mathrm{X} \mathrm{TBE}$ (Takara Bio, Inc.).

\section{Results}

In 8 patients with $\mathrm{CMN}$, there were 7 FRMD7 gene mutations (6 new mutations). The screening rate was $38.89 \%$, including c.41_43delAGA (p.13-15delK); c.473T >A (p.I158N); c.605T >A (p.I202N); c.580G $>$ T (p.A194S); c.811T $>A$ (p.C271S); c.1493insA (p.Y498X); c.57+1G>A (slice mutation; Fig. 2).

The mutation identified in the present study resulted in a change at the protein level with a residue substitution weight by Blosum 62, and a 'probable damaging' effect identified by PolyPhen (Table II). The conservation of the protein in this position was identified by analyzing seven orthologs from different mammalian species (Fig. 3). These mutations were also investigated in 96 unaffected control individuals (Fig. 4) by heteroduplex-SSCP, but none were identified.

In the current study, all patients with idiopathic nystagmus were male. Nystagmus usually manifests as a midrange decline in visual acuity. The uncorrected visual acuity ranged from 0.1 to 0.4 and corrected visual acuity ranged from 0.4 to 0.9 (Table III). The results of customary slit lamp and fundus examinations were normal.

\section{Discussion}

$\mathrm{CMN}$ is an ocular motor disorder characterized by involuntary oscillation of the eyes that occurs in the first 6 months of life. It is speculated that the disease may be associated with the abnormal development of senior center monitoring the abnormal eye movements and eye gazing. The prevalence of $\mathrm{CMN}$ in the population is estimated to be 24 per 10,000 (15). It is known to be genetically heterogeneous as autosomal dominant, autosomal recessive and X-linked patterns of inheritance. $\mathrm{X}$-linked inheritance with incomplete penetrance is the most common form (4-8). However, the majority of patients of CMN are sporadic cases.

Until now, FRMD7 is the only one gene that has been identified for X-linked CMN. It was demonstrated that FRMD7 expression is spatially and temporally regulated in the developing human embryonic cortex. FRMD7 is expressed in most adult human tissues, and has been detected in the developing neuroretina and regions of the embryonic brain known to control eye movement (16). FRMD7 consists of 12 exons and encodes a polypeptide with 714 residues polypeptide, spanning approximately $\sim 51 \mathrm{~kb}$ on chromosome Xq26-q27. The FRMD7 protein has B41, FERM-N, FERM-M and FERM-C 
Table I. Oligonucleotides used for FRMD7 amplification.

\begin{tabular}{|c|c|c|c|}
\hline Exon & Sequences $\left(5^{\prime}-3^{\prime}\right)$ & $\begin{array}{l}\text { Size of PCR } \\
\text { products (bp) }\end{array}$ & $\begin{array}{c}\text { Annealing } \\
\text { temperature }\left({ }^{\circ} \mathrm{C}\right)\end{array}$ \\
\hline FRMD7-exon1-f & AGGAAGTCCAGTTAGATTTG & 428 & 58.7 \\
\hline FRMD7-exon1-r & ACAGTCCTCCTTCATTCAGT & & \\
\hline FRMD7-exon2-f & ATGCAGGTCCTCTAAACAGT & 320 & 58.7 \\
\hline FRMD7-exon2-r & GGAATTGAACCCTACATACC & & \\
\hline FRMD7-exon3-f & GAAAATATAAGGGGGCAGAT & 368 & 54.4 \\
\hline FRMD7-exon3-r & TGGATGTATGAAGGGTTGAA & & \\
\hline FRMD7-exon4-f & GAGGGGACGGAAGAGGAGA & 287 & 61.6 \\
\hline FRMD7-exon4-r & TGAGAATGGCCAGAAGCACT & & \\
\hline FRMD7-exon5-f & CCCCAAAAAGGCATCTGA & 339 & 57.3 \\
\hline FRMD7-exon5-r & TCTCССCТGTAAACССТАAC & & \\
\hline FRMD7-exon6-f & GATGGAGGACAAGGGTATGC & 393 & 58.7 \\
\hline FRMD7-exon6-r & GCCACTGAAAGGGGAAAGAA & & \\
\hline FRMD7-exon7-f & AGCAAGCCCTTAAACCTGAG & 391 & 58.7 \\
\hline FRMD7-exon7-r & СССTTTCTGGCTGGTGATAA & & \\
\hline FRMD7-exon8-f & AATGCCTTCTTTGACCACAGC & 365 & 62.9 \\
\hline FRMD7-exon8-r & GCCAGCCGGCTTTTACAAT & & \\
\hline FRMD7-exon9-f & AGTGGCCCTGTCTATTCCTC & 551 & 62.9 \\
\hline FRMD7-exon9-r & GGTGCCCCCATCTTCCTC & & \\
\hline FRMD7-exon10, exon11-f & CTGCCTGGTCCTTGAATAAG & 582 & 57.3 \\
\hline FRMD7-exon10, exon11-r & TCCCCAGGAAGCTAACCTA & & \\
\hline FRMD7-exon12a-f & ATGGATCTTGTTAAATGACTT & 541 & 51.1 \\
\hline FRMD7-exon12a-r & ACCAACCTGCTGACCTGTA & & \\
\hline FRMD7-exon12b-f & TCCCACATTGCTACATCAGTC & 520 & 58.7 \\
\hline FRMD7-exon12b-r & CAAATTTGGGTCTTCCTCTTC & & \\
\hline FRMD7-exon12c-f & ATGTGCCCTATATTCCTTGTA & 592 & 58.7 \\
\hline FRMD7-exon12c-r & ATGGGTGACCTTATTTCTTTG & & \\
\hline FRMD7-exon12d-f & TCCCAGAGCCAATCAGACAT & 435 & 58.7 \\
\hline FRMD7-exon12d-r & TTTCTGCCTAAGTCGGTAACA & & \\
\hline $\begin{array}{l}\text { FRMD7-mutation } \\
\text { (c.1403G>A/c.1492-1493intA)-f }\end{array}$ & CAAGCTGTAAGTTTTCTGGTAATC & 213 & a \\
\hline $\begin{array}{l}\text { FRMD7-mutation } \\
\text { (c.1403G>A/c.1492-1493intA)-r }\end{array}$ & GACTTGTCCTTTCCTCTGCTC & & \\
\hline FRMD7-mutation (c.473T>A)-f & TGCTCCATTGCTAAGTTCCTCA & 234 & 56.8 \\
\hline FRMD7-mutation (c.473T>A)-r & TCTGTCCCCAATTTTAGTGTTCTC & & \\
\hline FRMD7-mutation (c.605T>A)-f & CATTCTTGAGGCATTTATTAGG & 173 & a \\
\hline FRMD7-mutation (c.605T>A)-r & GTGAGCAACAGCCAGGTGA & & \\
\hline FRMD7-mutation (c.580G>T)-f & GAGCTCTCAGGGTGGAAATGTCAT & 246 & 61.1 \\
\hline FRMD7-mutation (c.580G>T)-r & GCTGAAGGGCTTGAAAGGAA & & \\
\hline FRMD7-mutation (c.811T>A)-f & GTTTGGAAGGCATTGGGATTTGAA & 203 & 63.2 \\
\hline FRMD7-mutation (c.811T>A)-r & TTTGGGCTTTGATTTGGGCTCTT & & \\
\hline FRMD7-mutation (c.57+1G >A)-f & CCTCGCTGAGAATGCTAC & 189 & a \\
\hline FRMD7-mutation (c.57+1G>A)-r & ATCACAGTCCTCCTTCAT & & \\
\hline
\end{tabular}

aTouch down PCR program. PCR, polymerase chain reaction; FRMD7, FERM domain containing 7.

domains. The conserved domains are at the B41 and FERM-C domains. The B41 domain is located between residues 1-192, and the FERM-C domain is located between residues 186-279.

Many mutations in FRMD7 have been reported in Chinese families with CMN. In the current study, the authors attempted to identify FRMD7 mutations and investigate the clinical phenotype of sporadic cases with CMN in the Chinese population. In the present study, there were seven FRMD7 gene mutations, six of which 6 gene mutations are newly-identified in 7 patients with CMN. The screening 


\section{Touch-Down PCR}

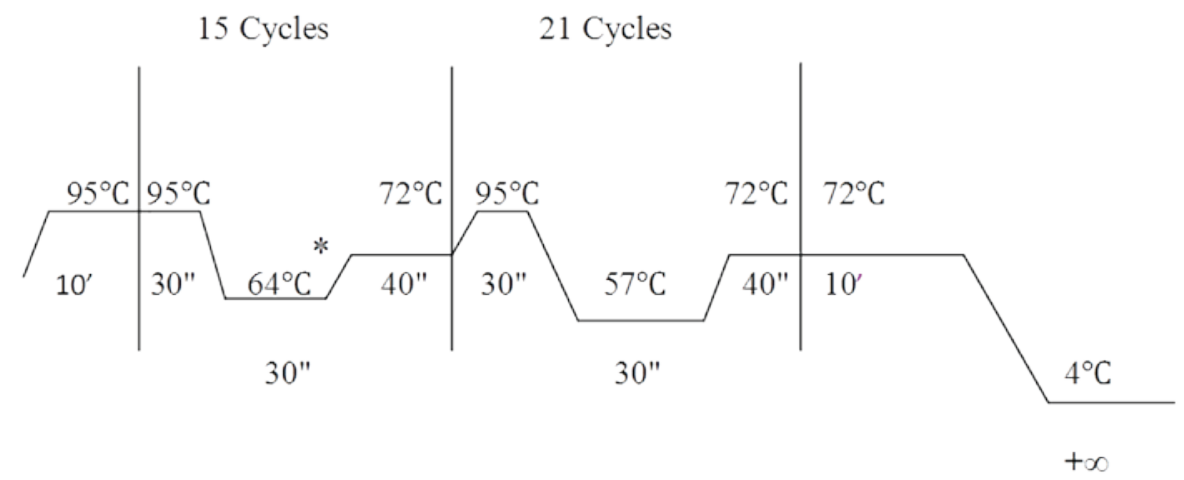

Figure 1. The touch down PCR program. ${ }^{*}$ Temperature drop of $0.5^{\circ} \mathrm{C}$ per cycle. PCR, polymerase chain reaction.

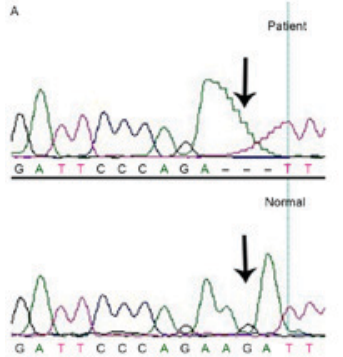

c
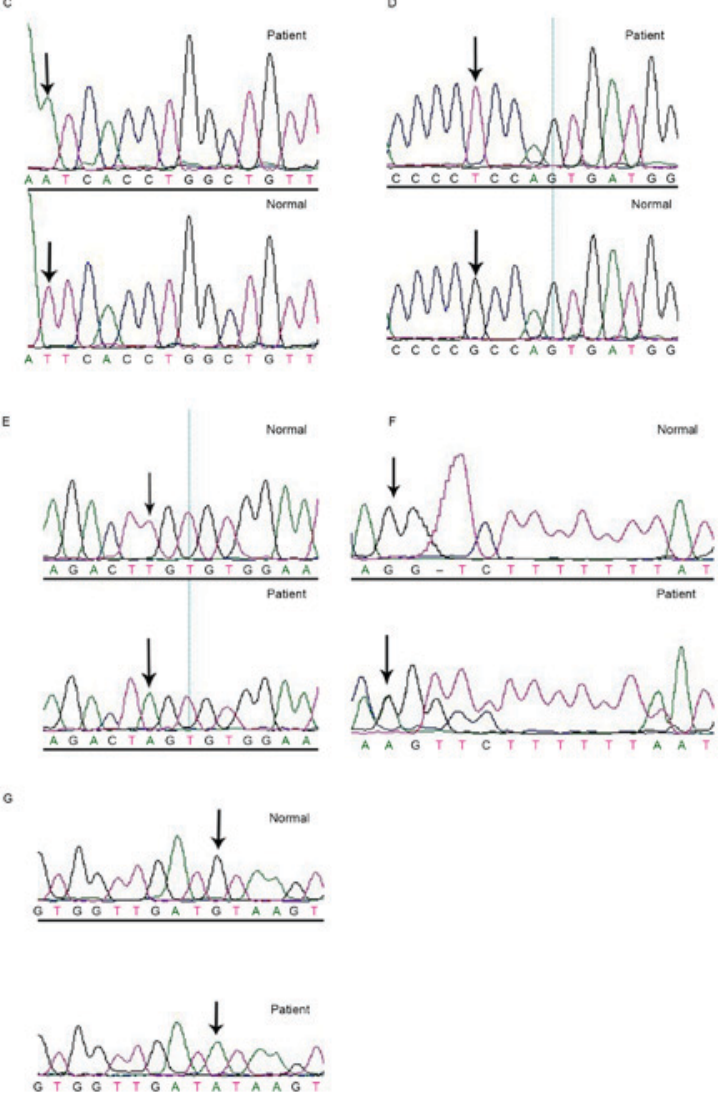

Figure 2. The map of FRMD7 direct sequencing. The mutations of (A) c.41_43delAG, (B) c.473T >A (p.I158N), (C) c.605T >A (p.I202N), (D) c.580G $>\mathrm{T}$ (p.A194S), (E) c.811T >A (p.C271S), (F) c.1492-1493insA (p.Y498X) and (G) c.57+1G>A were identified in the patient with congenital motor nystagmus and confirmed by bidirectional sequencing, which the arrow indicates. The other arrow shows the corresponding normal sequence from the unaffected control individual. rate is was $38.89 \%$, which is consistent with the previous studies $(17,18)$.

Through the MegAlign program, the protein sequences of different species were compared and mutation sites were identified (p.I158N, p.I202N, p.A194S and p.C271S), possessing relative conservation. Though further analysis of missense mutations, it was demonstrated that mutations of p.I158N, p.I202N and p.C271S mutations are possibly damaging with scores ranging from 0.997 to 0.998 . The mutation of p.A194S is was benign, but with a score of 0.993 .

FRMD7 is highly expressed in regions of the developing brain that are involved in oculomotor control, as well as in the retina (16). FRMD7 may serve a role in development of the oculomotor neural circuitry, previous studies indicate that nystagmus-associated mutations in the FERM and FA domains are likely to be critical to FRMD7 function (19). The mutations of c.41_43delAGA (p.13-15delK), c.473T>A (p.I158N), c.605T $>$ A (p.I202N), c.580G $>$ T (p.A194S) and c.811T >A (p.C271S) are in the conserved region of FRMD. Of them, the mutations of c.41_43delAGA (p.13-15delK), c.473T $>$ A (p.I158N), c.580G $>$ T (p.A194S) are in the B41 domain. The mutations of c.605T $>$ A (p.I202N) and c.811T $>$ A (p.C271S) are in the FERM-C domain. CMN-associated missense mutations within the $\mathrm{N}$-terminal region of the protein indicate that mutations can disrupt the interaction with other proteins, preventing their co-localization at the plasma membrane and impairing neurite formation (20).

The mutation site of p.Y498X is in the FERM-adjacent (FA) domain. FA is identified in a subset of FERM domain proteins, and which has been indicated to regulate protein function through modifications such as phosphorylation (21).

In summary, CMN is a genetically heterogeneous ocular movement disease. The presented result expands the mutation spectrum of FRMD7 and provides evidence for future functional studies, clinical diagnosis, differential diagnosis and genetic counseling. In conclusion, these results enriched the gene mutation spectrum of FRMD7. The authors systematically investigated the clinical phenotype of congenital motor nystagmus in the Chinese population, and provided further evidence for clinical diagnosis and differential diagnosis and genetic counseling. 
Table II. Analysis by Blosum62 and PolyPhen.

\begin{tabular}{lccll}
\hline Gene & Mutation & Blosum62 & \multicolumn{1}{c}{ PolyPhen } \\
\hline FRMD7 & I158N & $4 \rightarrow-3$ & Possibly damaging & 0.998 \\
FRMD7 & I202N & $4 \rightarrow-3$ & Possibly damaging & 0.998 \\
FRMD7 & A194S & $4 \rightarrow 1$ & Benign & 0.993 \\
FRMD7 & C271S & $9 \rightarrow-1$ & Possibly damaging & 0.997
\end{tabular}

FRMD7, FERM domain containing 7.

Table III. The visual acuity of patients with CMN.

\begin{tabular}{lccc}
\hline Patient & $\begin{array}{c}\text { Age } \\
\text { (years) }\end{array}$ & $\begin{array}{c}\text { Uncorrected } \\
\text { visual acuity }\end{array}$ & $\begin{array}{c}\text { Corrected } \\
\text { vision }\end{array}$ \\
\hline QT276 & 18 & $0.3^{+} / 0.5^{-}$ & $\mathrm{NA}$ \\
QT321 & 12 & $0.2 / 0.1$ & $0.4 / 0.4$ \\
QT370 & 0.3 & Perception of light & $\mathrm{ND}$ \\
QT385 & 28 & $0.7^{+} / 0.8$ & $0.7^{+} / 0.8$ \\
QT423 & 8 & $0.1 / 0.1$ & $0.3 / 0.3$ \\
QT439 & 12 & $1.0 / 0.7$ & $1.0 / 0.7$ \\
QT474 & 10 & $0.5 / 0.4^{+}$ & $0.7 / 0.8$ \\
QT552 & 3.5 & $0.25 / 0.2$ & $\mathrm{ND}$ \\
QT596 & 5 & $0.3 / 0.3$ & $0.4 / 0.4$ \\
QT664 & 3 & $0.4 / 0.4$ & $0.5 / 0.5$ \\
QT669 & 3.5 & $\mathrm{ND}$ & $\mathrm{ND}$ \\
QT684 & 5 & $0.3 / 0.4$ & $0.7 / 0.7^{+}$ \\
QT712 & 3 & Perception of light & $\mathrm{ND}$ \\
QT719 & 29 & $0.1 / 0.1$ & $0.9 / 0.9$ \\
QT726 & 8 & $0.5 / 0.5$ & $\mathrm{ND}$ \\
QT756 & 5.5 & $0.4^{+} / 0.4^{+}$ & $0.7 / 0.6$ \\
QT762 & 6 & $0.3 / 0.3$ & $0.4 / 0.4$ \\
QT835 & 1 & Perception of light & $\mathrm{ND}$ \\
\hline
\end{tabular}

ND, not determined; NA, not applicable; CMN, congenital motor nystagmus.

\section{A \\ Bos taurus \\ Equus caballus \\ Mus musculus
Rattus norvegicus \\ Rattus norvegicus
Homo sapiens \\ Macaca mulatta \\ Pongo abelii \\ C \\ Bos taurus \\ Equus caballus \\ Mus musculus \\ Rattus norvegicus \\ Homo sapiens \\ Macaca mula}

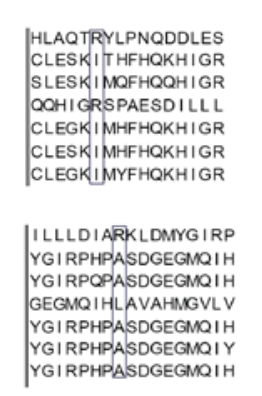

Bos taurus

Equus caballus

Mus musculus

Rattus norvegicus
Homo sapiens

Momo sapiens

Pongo abelii

Bos taurus

Equus caballus

Mus musculus

Rattus norvegicus

Homo sapiens

Macaca mulatta

Pongo abelii
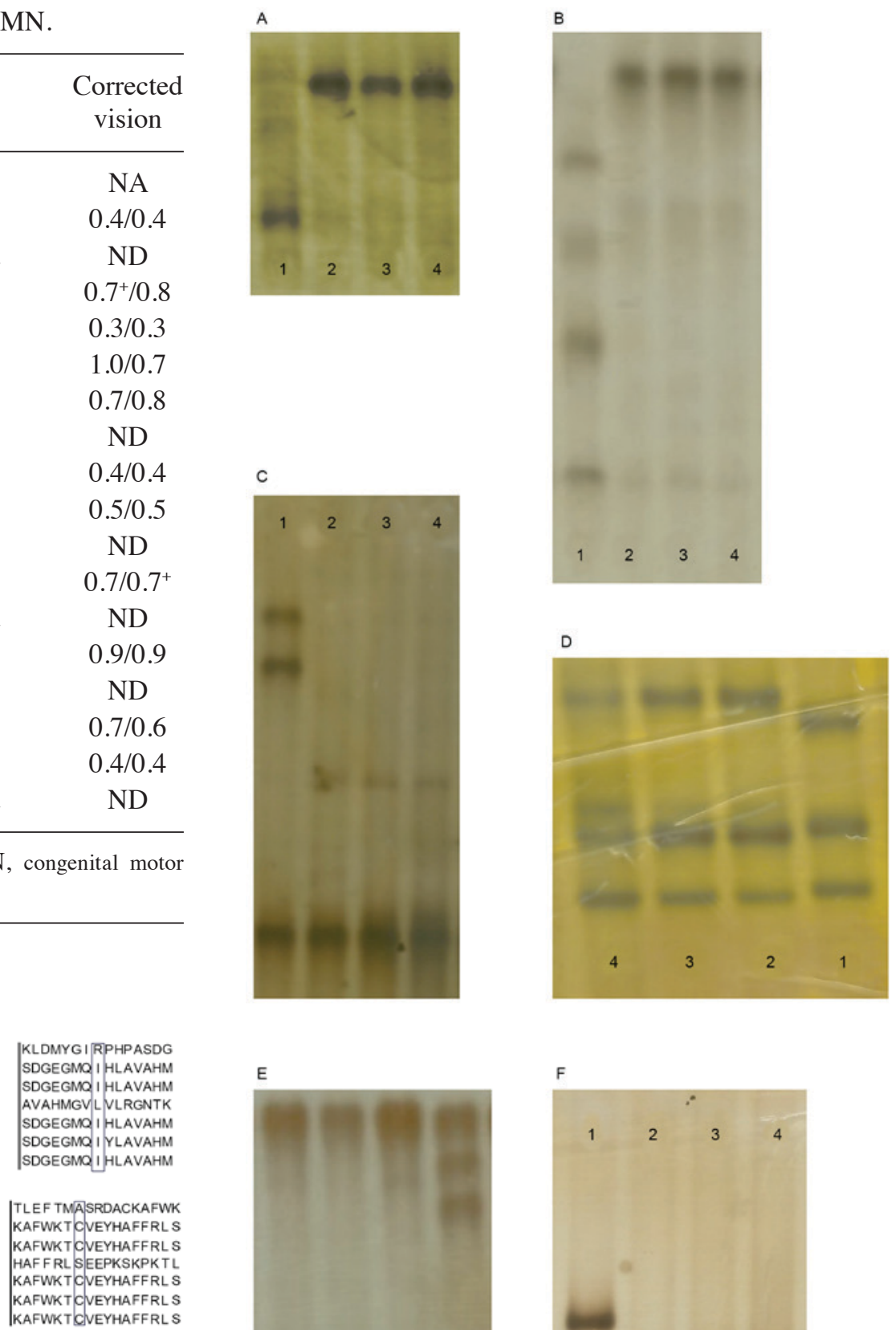

E
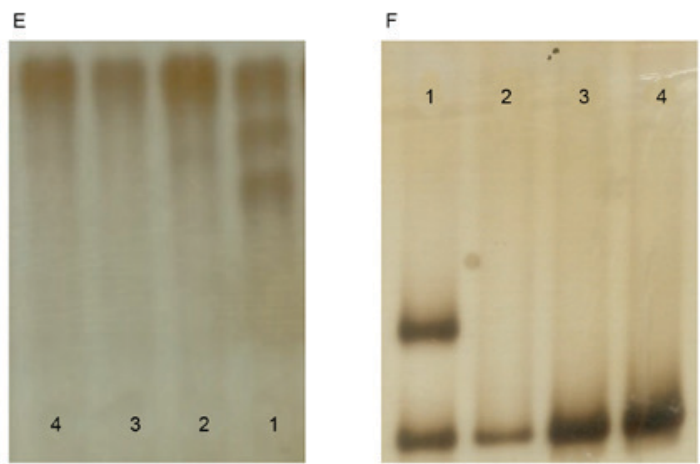

Figure 4. The HA-SSCP analysis of FRMD7 gene mutation in patients with CMN. Lane 1s indicate the bands of patients with mutation of (A) c.473T $>A$ (p.I158N), (B) c.605T >A (p.I202N), (C) c.580G >T (p.A194S), (D) c.811T >A (p.C271S), (E) c.1492-1493insA (p.Y498X) and (F) c.57+1G >A, and the other lanes show the bands of normal controls. 


\section{Acknowledgements}

The authors would like to thank the patients and family members for their participation. The present study was supported in part by the National Natural Science Foundation of China (grant no. 81170847).

\section{References}

1. Stayte M, Reeves B and Wortham C: Ocular and vision defects in preschool children. Br J Ophthalmol 77: 228-232, 1993.

2. Forssman B and Ringnér B: Prevalence and inheritance of congenital nystagmus in a Swedish population. Ann Hum Genet 35: 139-147, 1971.

3. Jacobs JB and Dell'Osso LF: Congenital nystagmus: Hypotheses for its genesis and complex waveforms within a behavioral ocular motor system model. J Vis 4: 604-625, 2004

4. Tarpey P, Thomas S, Sarvananthan N, Mallya U, Lisgo S, Talbot CJ, Roberts EO, Awan M, Surendran M, McLean RJ, et al: Mutations in FRMD7, a newly identified member of the FERM family, cause X-linked idiopathic congenital nystagmus. Nat Genet 38: 1242-1244, 2006.

5. Schorderet DF, Tiab L, Gaillard MC, Lorenz B, Klainguti G, Kerrison JB, Traboulsi EI and Munier FL: Novel mutations in FRMD7 in X-linked congenital nystagmus. Mutation in brief \#963. Online. Hum Mutat 28: 525, 2007.

6. Self JE, Shawkat F, Malpas CT, Thomas NS, Harris CM, Hodgkins PR, Chen X, Trump D and Lotery AJ: Allelic variation of the FRMD7 gene in congenital idiopathic nystagmus. Arch Ophthalmol 125: 1255-1263, 2007.

7. Zhang B, Liu Z, Zhao G, Xie X, Yin X, Hu Z, Xu S, Li Q, Song F, Tian J, et al: Novel mutations of the FRMD7 gene in X-linked congenital motor nystagmus. Mol Vis 13: 1674-1679, 2007.

8. Zhang Q, Xiao X, Li S and Guo X: FRMD7 mutations in Chinese families with X-linked congenital motor nystagmus. Mol Vis 13: 1375-1378, 2007.

9. Kerrison JB, Vagefi MR, Barmada MM and Maumenee IH: Congenital motor nystagmus linked to Xq26-q27. Am J Hum Genet 64: 600-607, 1999.

10. Cabot A, Rozet JM, Gerber S, Perrault I, Ducroq D, Smahi A, Souied E, Munnich A and Kaplan J: A gene for X-linked idiopathic congenital nystagmus (NYS1) maps to chromosome Xp11.4-p11.3. Am J Hum Genet 64: 1141-1146, 1999.
11. Adzhubei IA, Schmidt S, Peshkin L, Ramensky VE, Gerasimova A, Bork P, Kondrashov AS and Sunyaev SR: A method and server for predicting damaging missense mutations. Nat Methods 7: 248-249, 2010.

12. Sale MM, Craig JE, Charlesworth JC, FitzGerald LM, Hanson IM, Dickinson JL, Matthews SJ, Heyningen Vv Vv, Fingert JH and Mackey DA: Broad phenotypic variability in a single pedigree with a novel 1410delC mutation in the PST domain of the PAX6 gene. Hum Mutat 20: 322, 2002.

13. Stone EM: Leber congenital amaurosis-a model for efficient genetic testing of heterogeneous disorders: LXIV Edward Jackson Memorial Lecture. Am J Ophthalmol 144: 791-811, 2007.

14. Zhang Q and Minoda K: Detection of congenital color vision defects using heteroduplex-SSCP analysis. Jpn J Ophthalmol 40: 79-85, 1996.

15. Sarvananthan N, Surendran M, Roberts EO, Jain S, Thomas S, Shah N, Proudlock FA, Thompson JR, McLean RJ, Degg C, et al: The prevalence of nystagmus: The Leicestershire nystagmus survey. Invest Ophthalmol Vis Sci 50: 5201-5206, 2009.

16. Li N, Wang X, Wang Y, Wang L, Ying M, Han R, Liu Y and Zhao K: Investigation of the gene mutations in two Chinese families with X-linked infantile nystagmus. Mol Vis 17: 461-468, 2011.

17. Liu JY, Ren X, Yang X, Guo T, Yao Q, Li L, Dai X, Zhang M, Wang L, Liu M and Wang QK: Identification of a novel GPR143 mutation in a large Chinese family with congenital nystagmus as the most prominent and consistent manifestation. J Hum Genet 52: 565-570, 2007.

18. Zhang X, Ge X, Yu Y, Zhang Y, Wu Y, Luan Y, Sun J, Qu J, Jin $\mathrm{ZB}$ and $\mathrm{Gu} F$ : Identification of three novel mutations in the FRMD7 gene for X-linked idiopathic congenital nystagmus. Sci Rep 4: 3745, 2014.

19. Watkins RJ, Thomas MG, Talbot CJ, Gottlob I and Shackleton S: The role of FRMD7 in idiopathic infantile nystagmus. J Ophthalmol 2012: 460956, 2012.

20. Koyano Y, Kawamoto T, Shen M, Yan W, Noshiro M, Fujii K and Kato Y: Molecular cloning and characterization of CDEP, a novel human protein containing the ezrin-like domain of the band 4.1 superfamily and the Dbl homology domain of Rho guanine nucleotide exchange factors. Biochem Biophys Res Commun 241: 369-375, 1997.

21. Baines AJ: A FERM-adjacent (FA) region defines a subset of the 4.1 superfamily and is a potential regulator of FERM domain function. BMC Genomics 7: 85, 2006. 